\title{
Aging of the placenta
}

Harold Fox

\section{Introduction}

It is widely believed that during the relatively short duration of a normal pregnancy the placenta progressively ages and is, at term, on the verge of a decline into morphological and physiological senescence..$^{1-3}$ This belief is based on the apparent convergence of clinical, structural, and functional data, all of which have been taken, rather uncritically, as supporting this concept of the placenta as an aging organ with, all too often, no distinction being made between time related changes and true aging changes. I will review some of these concepts and consider whether the placenta truly undergoes an aging process. For the purposes of this review an aging change is considered to be one which is intrinsic, detrimental, and progressive and which results in an irreversible loss of functional capacity, an impaired ability to maintain homeostasis, and decreased ability to repair damage.

\section{Morphological changes}

The placenta is unusual in so far as its basic histological structure undergoes a considerable change throughout its lifespan. For some time it has been customary to describe the appearances of the placental villi in terms of their changing appearance as pregnancy progresses, comparing, for instance, typical first trimester villi with those in third trimester placentas. It has often been implied that this changing appearance is an aging process, but it is now recognised that this temporal variability in villous appearances reflects the continual development and branching of the villous tree (fig 1) In recent years the relation between the growth of the villous tree and the villous histological appearances has been formally codified ${ }^{5-8}$ with identification of five types of villi (fig 2 ).

\section{Mesenchymal villi}

These represent a transient stage in placental development and they can differentiate into either mature or immature intermediate villi. They comprise the first generation of newly formed villi and are derived from trophoblastic sprouts by mesenchymal invasion and vascularisation. They are found mainly in the early stages of pregnancy but a few may still be found at term They have complete trophoblastic mantles with many cytotrophoblastic cells and regularly dispersed nuclei in the syncytiotrophoblast: their loose, immature-type stroma is abundant and contains a few Hobauer cells, together with poorly developed fetal capillaries.

\section{Immature intermediate villi}

These are peripheral extensions of the stem villi and are the predominant form seen in immature placentas. These villi have a well preserved trophoblastic mantle in which cytotrophoblastic cells are numerous; the syncytial nuclei are evenly dispersed and there are no syncytial knots or vasculo-syncytial membranes. They have an abundant loose stroma that contains many Hofbauer cells: capillaries, arterioles, and venules are present.

\section{Stem villi}

These comprise the primary stems which connect the villous tree to the chorionic plate, up to four generations of short thick branches and further generations of dichotomous branches. Their principal role is to serve as a scaffolding for the peripheral villous tree, and up to one third of the total volume of the villous tissue of the mature placenta is made up of this villous type, the proportion of such villi being highest in the central subchorial portion of the villous tree. Histologically, the stem villi have a compact stroma and contain either arteries and veins or arterioles and venules; superficially located capillaries may also be present.

\section{Mature intermediate villi}

These are the peripheral ramifications of the villous stems from which most terminal villi directly arise. They are large $(60-150 \mu \mathrm{m}$ in diameter) and contain capillaries admixed with small arterioles and venules, the vessels being set in a very loose stroma which occupies more than half of the villous volume. The syncytiotrophoblast has a uniform structure, no knots or vasculo-syncytial membranes being present. Up to a quarter of the villi in a mature placenta are of this type.

\section{Terminal villi}

These are the final ramifications of the villous tree and are grape-like outgrowths from mature intermediate villi. They contain capillaries, many of which are sinusoidally dilated to occupy most of the cross sectional diameter of the villus. The syncytiotrophoblast is thin and the syncytial nuclei are irregularly dispersed. Syncytial knots may be present and vasculosyncytial membranes are commonly seen. These terminal villi begin to appear at about the 27 th week of gestation and account for 30-40 per cent of the villous volume, 50 per cent of the villous surface area, and 60 per cent of villi seen in cross section at term.

The pattern of development of the villous tree is therefore as follows: During the early weeks of pregnancy all the villi are of the mesenchymal type. Between the 7th and 8th weeks mesenchymal villi begin to transform into immature intermediate villi and these subsequently 


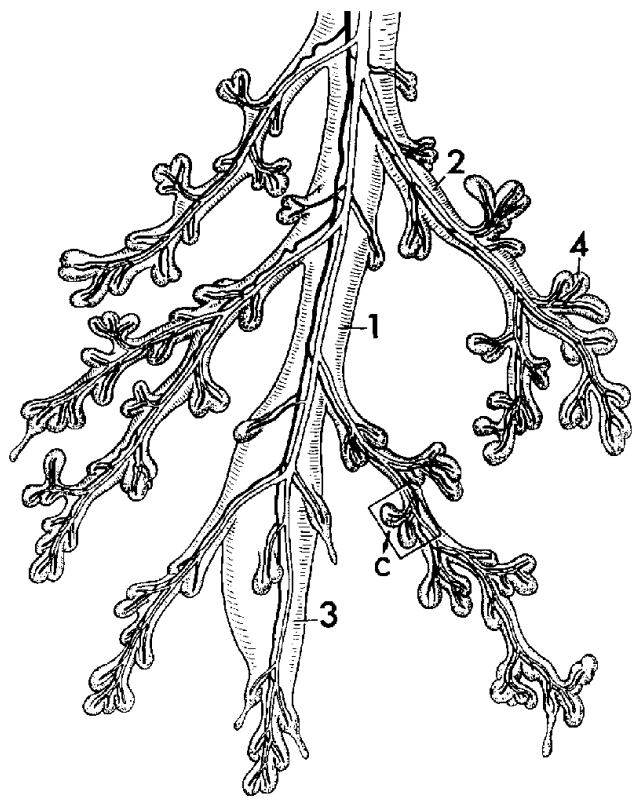

Figure 1 Diagrammatic representation of a peripheral villous tree, showing a large central stem villus: the lateral branches from this are the mature intermediate villi from which the terminal villi protrude.

transform into stem villi. Development of additional immature intermediate villi from mesenchymal villi gradually ceases at the end of the second trimester, but these immature intermediate villi continue to mature into stem villi and only a few persist to term as growth zones in the centres of the lobules. At the beginning of the third trimester mesenchymal villi stop transforming into immature intermediate villi and start transforming into mature

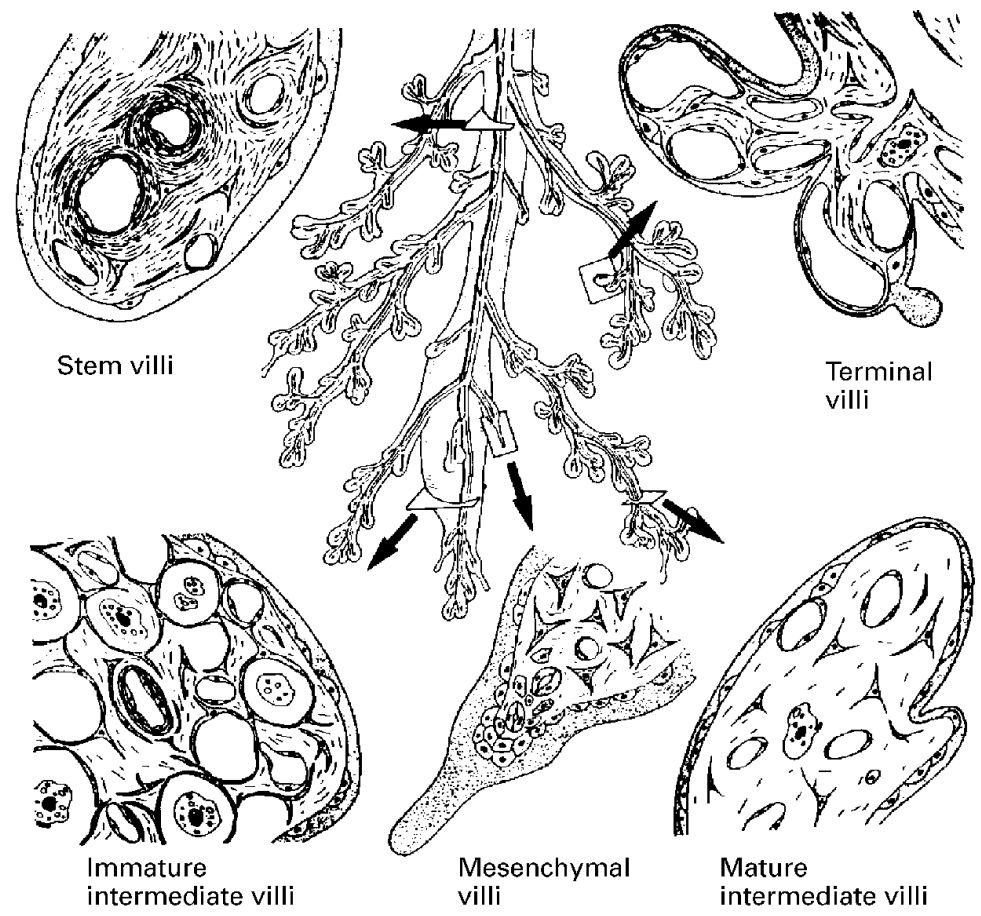

Figure 2 Representation of the peripheral branches of a mature villous tree together with typical cross sections of the five villous types. The figures are reproduced from Haines $\mathcal{E}$ Taylor. Textbook of Obstetrical and Gynaecological Pathology. 4th Edn. 1995, by kind permission of Churchill Livingstone and Professor P Kaufmann. intermediate villi. The latter serve as a framework for the terminal villi which begin to appear shortly afterwards and predominate at term.

This progressive elaboration of the villous tree results in a predominance of terminal villi in the mature placenta. Such villi have been conventionally classed as "third trimester villi" and a comparison of their structure with the predominant type of villi in the first trimesterimmature intermediate villi-has led many to suggest that as pregnancy progresses the villous trophoblast becomes irregularly thinned and the cytotrophoblast regresses, changes interpreted as being of an aging nature. The villous cytotrophoblast, which is a stem cell for the trophoblast, does not in reality regress, because the absolute number of these cells in the placenta is not decreased at term and in fact continues to increase throughout pregnancy. The apparent sparsity of these cells is due to their wider dispersion within a greatly increased total placental mass. ${ }^{9}{ }^{10}$ The focal thinning of the villous syncytiotrophoblast apparent in many terminal villi has often been cited as evidence of syncytial senescence. These thinned areas are, in reality, the "vasculosyncytial membranes"11 which, although formed in part by mechanical stretching of the trophoblast by ballooning capillary loops, ${ }^{12}$ never the less differ enzymatically and ultrastructurally from the non-membranous areas of the syncytium and are areas of the syncytiotrophoblast specifically differentiated for the facilitation of gas transfer. ${ }^{13}$ These membranes are therefore a manifestation of topographic functional differentiation within the trophoblast.

The interlinked, but separate, processes of maturation of the villous tree and functional differentiation of the trophoblast result in a predominant villous form that is optimally adapted for materno-fetal transfer diffusion mechanisms: the morphological changes substantially increase trophoblastic surface area ${ }^{14}$ and a significantly reduce the harmonic mean of the diffusion distance between maternal and fetal blood, ${ }^{15}$ with a resulting increase in the conductance of oxygen diffusion. ${ }^{16}$

It is not mere pedantry to distinguish between maturation, which results in increased functional efficiency, and aging, which results in decreased functional efficiency. In this respect it is worth noting that a proportion of placentas from women with severe preeclampsia look unusually mature for the length of the period of gestation: this is usually classed as "premature aging" but it would be more accurate to regard the changes as being due to accelerated maturation, this being a compensatory mechanism to increase the transfer capacity of the placenta in the face of an adverse maternal environment.

It has to be admitted that the control mechanisms of placental maturation are unknown. There are many agents thought to be of importance in the control of placental growth, including cytokines, growth factors, oncogenes, prostaglandins and leucotrienes, ${ }^{17-20}$ but it far from clear as to whether control of growth 
can be equated with control of maturation. However, villous development, certainly in the later stages of pregnancy, does seem to be driven principally by proliferation of endothelial cells and capillary growth. ${ }^{21}$ Vascular endothelial growth factors are present in placental tissue ${ }^{22}$ and the suggestion that hypoxia may stimulate angiogenesis, ${ }^{23}$ and thus have a significant role in placental development, would corroborate the accelerated placental maturation seen in some cases of maternal pre-eclampsia.

\section{Placental growth}

It has long been maintained that placental growth and DNA synthesis cease at about the 36 th week of gestation and that any subsequent increase in placental size is due to an increase in cell size rather than to an increase in the number of cells. ${ }^{24}$ Simple histological examination of the term placenta will, however, serve to refute this view, because immature intermediate villi are often present in the centres of lobules and these clearly represent a persistent growth zone. Furthermore, total placental DNA content continues to increase in an almost linear manner until and beyond the $42 \mathrm{nd}$ week of gestation. ${ }^{25}$ This finding agrees with autoradiographic and flow cytometric studies that have shown continuing DNA synthesis in the term organ, ${ }^{26-28}$ and with morphometric investigations that have shown persistent villous growth, continuing expansion of the villous surface area, and progressive branching of the villous tree up to and beyond term. ${ }^{1429}$

Placental growth certainly slows, but clearly does not cease, during the last few weeks of gestation, although this decline in growth rate is neither invariable nor irreversible, because the placenta can continue to increase in size if faced with an unfavourable maternal environment, such as pregnancy at high altitude, or severe maternal anaemia, while the potential for a recrudescence of growth is shown by the proliferative response to ischaemic syncytial damage. Those who contend that a decreased placental growth rate during late pregnancy is evidence of senescence often seem be comparing the placenta with an organ such as the gut, in which continuing viability depends on a constantly replicating stem cell layer producing short-lived postmitotic cells. A more apt comparison would be with an organ such as the liver, which is formed principally of long-lived postmitotic cells and which, once an optimal size has been attained to meet the metabolic demands placed on it, shows little evidence of cell proliferation while retaining a latent capacity for growth activity. There seems no good reason why the placenta, once it has reached a size sufficient to adequately meet its transfer function, should continue to grow, and the term placenta, with its considerable functional reserve capacity, has more than met this aim.

\section{Functional activity}

There have been few vertical studies of placental function throughout pregnancy, but there is no evidence that any of the major indices of placental function decline-namely, proliferative, transfer, and secretory capacities. ${ }^{30}$ As already remarked, the diffusion conductance of the organ is increased, largely as a result of morphological changes, but there is considerable evidence that specific placental carrier mediated transfer systems are also augmented. ${ }^{20}$ The placental production of hormones continues unabated until term: the synthesis of human chorionic gonadotrophin declines towards the end of the first trimester but this is clearly due to a gene switch which results in progressively increasing secretion of human placental lactogen.

The placenta also retains its full proliferative capacity until term as shown by its ability to repair and replace, as a result of proliferation in the villous cytotrophoblastic cells, of a villous syncytiotrophoblast that has been ischaemically damaged in women with severe pre-eclampsia. ${ }^{13}$

\section{Clinical factors}

The single most important factor leading to a belief in placental senescence has been the apparently increased fetal morbidity and mortality associated with prolonged pregnancy, this traditionally being attributed to "placental insufficiency" consequent on senescence of the organ. ${ }^{131}$ In the past it was thought that about $11 \%$ of pregnancies extended to or beyond the 42 nd week of gestation ${ }^{32} 33$ : the introduction of a routine ultrasound examination in early pregnancy reduced the incidence of prolonged pregnancies to about $6 \%{ }^{34}$ and it has even been claimed that with very accurate dating studies the incidence of truly prolonged gestations does not exceed $1 \% .^{35}$ This casts some doubt on the validity of a great deal of the historical information about the risks and ill effects of prolonged pregnancy, but it is never the less widely accepted that perinatal mortality increases after the 42nd week of gestation. $^{36}$

Any increase in perinatal mortality after the $42 \mathrm{nd}$ week of gestation is due, in part, to the high incidence of fetal macrosomia: $10 \%$ of infants from prolonged pregnancies weigh over $4000 \mathrm{~g}$ and $1 \%$ over $4500 \mathrm{~g}$ and these fetuses are at particular risk of complications such as shoulder dystocia. The presence of this large number of macrosomic fetuses is a clear indication that, in this subset at least, the placenta continues to function well beyond the 40 th week of gestation and remains capable of sustaining untrammelled fetal growth.

The classic clinical syndrome of the "postmature" infant ${ }^{131}$ is not commonly seen today but seems to be clearly related to the development of oligohydramnios. There is no doubt that amniotic fluid volume tends to decrease in a proportion of prolonged pregnancies ${ }^{39}$ and that oligohydramnios is associated with a high incidence of fetal heart rate decelerations. ${ }^{36}$ This has been attributed by some to cord compression, ${ }^{40}{ }^{41}$ but one study, while confirming that cord compression is common in prolonged pregnancies, was unable to correlate 
such compression with fetal acidosis. ${ }^{42}$ It is often assumed, and indeed commonly stated, that the decline in amniotic fluid volume in these cases is an indication of "placental insufficiency" but, in reality, there is no evidence that in late pregnancy the placenta plays any part in the production of amniotic fluid or in the control of amniotic fluid volume. ${ }^{43}$

The two most potent causes of increased morbidity in prolonged pregnancy are therefore clearly unrelated to any change in placental functional capacity. Examination of placentas from prolonged pregnancies shows no evidence of any increased incidence of gross placental abnormalities, such as infarcts, calcification, or massive perivillous fibrin deposition. The most characteristic histological abnormality, found in a proportion of cases but certainly not in all, is decreased fetal perfusion of the placental villi. ${ }^{13}$ The fetal villous vessels are normal in placentas from prolonged pregnancies $^{44}$ and Doppler flow velocimetry studies have, in general but not unanimously, indicated that there is no increased fetal vascular resistance in such placentas. ${ }^{45-47}$ The decreased fetal perfusion is therefore probably a consequence of oligohydramnios, because umbilical vein flow studies have shown that fetal blood flow to the placenta is often reduced in cases of oligohydramnios. ${ }^{48}$

It has to be admitted that the pathophysiology of prolonged pregnancy has not been fully elucidated. It seems, however, quite clear that any ill effects which may befall the fetus in prolonged gestations can not be attributed to placental insufficiency or senescence.

\section{Conclusions}

A review of the available evidence indicates that the placenta does not undergo a true aging change during pregnancy. There is, in fact, no logical reason for believing that the placenta, which is a fetal organ, should age while the other fetal organs do not: the situation in which an individual organ ages within an organism that is not aged is one which does not occur in any biological system. The persisting belief in placental aging has been based on a confusion between morphological maturation and differentiation and aging, a failure to appreciate the functional resources of the organ, and an uncritical acceptance of the overly facile concept of "placental insufficiency" as a cause of increased perinatal mortality.

1 Vorherr H. Placental insufficiency in relation to to postterm pregnancy and fetal postmaturity: evaluation of fetoplacental function: management of the postterm gravida. $A m \mathcal{F}$ Obstet Gynecol 1975;123:67-103.

2 Vincent RA, Huang PC, Parmley TH. Proliferative capacity of cell cultures derived from the human placenta. In Vitro 1976;32:649-53.

3 Rosso P. Plaenta as an ageing organ. Curr Concept Nutr 1976;4:23-41

4 Kaufmann P. Development and differentiation of the human placental villous tree, Bibl Anat 1982;22:29-39.

5 Kaufmann P, Sen DK, Schweikhart G. Classification of human placental villi. I. Histology and scanning electron microscopy. Cell Tissue Res 1979;200:409-23.

6 Sen DK, Kaufmann P, Schweikhart. Classification of human placental villi.II. Morphometry. Cell Tissue Res 1979;200:425-34.
7 Castelluchi M, Scheper M, Scheffen I, Calona A, Kaufmann P. The development of the human placental villous tree. P. The development of the hum

8 Kosanke G, Castelluchi M, Kaufmann P, Minirov VA. Branching patterns of human placental villous tree: perpectives of topological analysis. Placenta 1993;14:591604

9 Simpson RA, Mayhew TM, Barnes PR. From 13 weeks to term, the trophoblast of human placenta grows by the continuous recruitment of new proliferative units: a study of nuclear number using the dissector. Placenta 1992;13:501-12.

10 Mayhew TM, Simpson RA. Quantitative evidence for the spatial dispersal of trophoblast nuclei in human placental villi during gestation. Placenta 1994;15:837-44.

11 Getzowa S, Sadowsky A. On the structure of the human placenta with full term and immature foetus, Living or placenta with full term and immature foetus, Livin

12 Burton GJ, Tham SW. The formation of vasculosyncytial membranes in the human placenta. $f$ Devel Physiol 1992;18:43-7.

13 Fox H. Pathology of the placenta. 2nd edn. London:WB Saunders, 1997

14 Boyd PA. Quantitative studies of the normal human placenta from 10 weeks of gestation to term. Early Hum Devel 1984;9:297-307.

15 Fenley MR, Burton GJ. Villous composition and membrane thickness in the human placenta at term: a stereological study using unbiased eximators and optimal fixation techniques. Placenta 1991;12:131-42.

16 Mayhew TM, Jackson MR, Boyd PA. Changes in oxygen diffusive conductances of human placentae during gestadiffusive conductances of human placentae during gesta-
tion $(10-41$ weeks) are commensurate with the gain in fetal weight. Placenta 1993;14:51-61.

17 Ohlsson R. Growth factors, protooncogenes and human placental development. Cellular Differential Development 1989;28:1-16.

18 Ohlsson R, Glaer A, Holmgren L, Franklin G. The molecular biology of placental development. In: Redman CWG, Sargent IL, Starkey PM, eds. The human placenta. Oxford: Blackwells, 1993: 33-81.

19 Mitchell MD, Trautman DS, Dudley DJ. Cytokine networking in the placenta. Placenta 1993; 14:249-75.

20 Schneider $\mathrm{H}$. Ontogenic changes in the nutritive function of the placenta. Placenta 1996;17: 15-26.

21 Kaufmann P, Luckhardt M, Leiser R. Three dimensional representation of the fetal vessel system in the human plarepresentation of the fetal vessel
centa. Troph Res 1988;3:113-37.

22 Anthony FW, Wheeler T, Elcock CL, et al. Identification of specific pattern of VEGF mRNA expression in human placenta and cultured placental fibroblast. Placenta 1994;15:561-2.

23 Wheeler T, Elcock CL, Anthony FW. Angiogenesis and the placental environement. Placenta 1995;16:289-96.

24 Winick M, Coscia A, Noble A. Cellular growth in human placenta. 1. Normal cellular growth. Pediatrics

25 Sands J, Dobbing J. Continuing growth and development of third trimester human placenta. Placenta 1985;6:13-22.

26 Geier G, Schuhmann R, Kraus H. Regionale unterschliedliche Zellproliferation innerhalb der Plazentome reifer menschlicher Plazenten: autoradiographische Untersucmenschlicher Plazenten: autoradiograph

27 Hustin J, Foedart JM, Lambotte R. Cellular proliferation in villi of normal and pathological pregnancies. Gynecol Obstet Invest 1984;17:1-9.

28 Iverson IE, Farsund T. Flow cytometry in the assessment of human placental growth. Acta Obstet Gynecol Scand 1985;64:605-7.

29 Jackson MR, Mayhew TM, Boyd PA. A quantitative description of the elaboration and maturation of villi from 10 weeks of gestation to term. Placenta 1992;13:357-70.

30 Fox H. The placenta as a model for organ aging. In: Beaconsfield P, Villee C, eds. Placenta-a neglected experimental animal. Oxford: Pergamon, 1993: 351-78.

31 Voherr H. Placental insuffficiency and postmaturity. Eur $\mathcal{F}$ Obstet Gynecol Reprod Biol 1975; 5:109-22.

32 Bolte A, Bachmann KD, Hofmann E, Rohricht J, Strothmann G. Verlangerte Schwangerschaftsdauer und Placentadysfunktion. I. Haufigkeit und und Diagnostik bei den Geburtsjahrgangen 1955-1996. Deutsche Med Wochenschrift 1972;97:671-5.

33 Perkins RP. Antenatal assessment of fetal maturity: a review. Obstet Gynecol Surv 1974; 29:369-84.

34 Cardozo L, Fysh J, Pearce JM. Prolonged pregnancy: the management debate. BM7 1986;293: 1059-63.

35 Boyd ME, Usher RH, McLean FH, Kramer MS. Obstetric consequences of post maturity. Am $\mathcal{f}$ Obstet Gynecol 1988;157:334-8.

36 Cardozo L. Prolonged pregnancy. In: Chamberlain G, ed. Turnbull's Obstetrics, 2nd edn. Edinburgh: Churchill Livingstone, 1995:633-43.

37 Spellacy WNG, Miller MS, Winegar A, et al. Macrosomia: maternal characteristics and infant complications. Obstet Gynecol 1985;66:158-61.

38 Modanlou HK, Dorchester WL, Thorosian A, Freeman RK. Macrosomia: maternal, fetal and neonatal considerations. Obstet Gynecol 1980;55:420-3.

39 Phelan JP, Smith CV, Broussard P, Sucell M. Amniotic fluid volume assessment with the four quadrant technique at volume assessment with the four quadrant technique

40 Gabbe SG, Ettinger BB, Freeman RK, Makrhn CB. Umbilical cord compression associated with amniotomy: 
laboratory observations. $A m$ f Obstet Gynecol 1977;126:353-5.

41 Leveno KJ, Quirk JG Jnr, Cunningham FG, et al. Prolonged pregnancy. I. Observations concerning the causes of fetal distress. Am 7 Obstet Gynecol 1984:150:465-73.

42 Silver RK, Dooley SJ, MacGregor SN, Depp R. Fetal acidosis in prolonged pregnancy cannot be atributed to cord compression alone. Am f Obstet Gynecol 1988;159:666-9.

43 Hytten F. Physiology and pathology of amniotic fluid formation. In: Fox H, ed. Haines and Taylor: obstetrical and gynaecological pathology. 3rd edn. Edinburgh: Churchill Livingstone, 1987: 1177-82.

44 Larsen LG, Clausen HV, Anderson B, Graem N. A stereologic study of postmature placentas fixed by dual perfusion. Am f Obstet Gynecol 1995;175:500-7.
45 Guidetti DA, Divon MY, Cavalieri RL, Langer O, Merkatz IR. Fetal umbilical artery flow velocimetry in postdate pregnancies. Am f Obstet Gynecol 1987;157:1521-3.

46 Stokes HJ, Roberts RV, Newnham JP. Doppler flow waveform velocity analysis in post-date pregnancies. Aust NZ 7 Obstet Gynaecol 1991;31:27-30.

47 Zimmermann P, Alback T, Koskinen J, et al. Doppler flow velocimetry of the umbilical artery, uteroplacental arteries and fetal middle cerebral artery in prolonged pregnancy. Ultrasound Obstet Gynecol 1995;5:189-97.

48 Gill RW, Warren PS, Garrett WJ, Kossoff G, Stewart A. Umbilical vein blood flow. In: Chervenack FA, Isaacson GC, Campbell S. eds. Ultrasound in obstetrics and gynecology. Boston: Little, Brown, 1993:587-95. 